\title{
Case detection and diagnosis of tuberculosis in primary-care settings
}

\section{S. M. Graham \& M. P. Sekadde}

To cite this article: S. M. Graham \& M. P. Sekadde (2019): Case detection and diagnosis of tuberculosis in primary-care settings, Paediatrics and International Child Health, DOI: 10.1080/20469047.2019.1597487

To link to this article: https://doi.org/10.1080/20469047.2019.1597487

曲 Published online: 08 Apr 2019.

Submit your article to this journal

View Crossmark data 


\title{
Case detection and diagnosis of tuberculosis in primary-care settings
}

\author{
S. M. Graham (D) and M. P. Sekadde ${ }^{\mathrm{a}, \mathrm{c}}$ \\ ${ }^{a}$ Centre for International Child Health, University of Melbourne and Murdoch Childrens Research Institute, Royal Childrens Hospital, \\ Melbourne, Australia; 'International Union Against Tuberculosis and Lung Disease, Paris, France; 'National Tuberculosis and Leprosy \\ Programme, Kampala, Uganda
}

ARTICLE HISTORY Received 5 March 2019; Accepted 16 March 2019

KEYWORDS Primary care; child tuberculosis; decentralisation; diagnostics evaluation; Xpert MTB/RIF

The World Health Organization (WHO) estimates that around 1 million children ( $<15$ years) develop tuberculosis (TB) annually [1]. Recent mortality estimates from modelling are that 239,000 children died of TB in 2015, that the majority $(80 \%)$ of these deaths occurred in infants and young children ( $<5$ years) and that more than $96 \%$ of the deaths occurred in children not detected with or treated for TB [2]. It is well recognised that young age is associated with an increased risk of disease following infection as well as an increased risk of severe disease and mortality compared with school-age children $[3,4]$. While young children make a minimal contribution to ongoing transmission of TB in the community, TB is an important treatable and preventable cause of under-5 morbidity and mortality [5]. It is less well recognised that TB is also a cause of life-long disability, for example, as a consequence of TB meningitis or spinal TB or severe pulmonary TB, especially if not detected and treated early [6].

Improving case detection, diagnostic accuracy and treatment availability are clearly critical to reduce TBrelated child morbidity and mortality [7]. However, important challenges for health services in providing these are common and inter-related. There is epidemiological overlap between areas where TB is endemic and under-5 mortality high which are also usually resourcelimited. TB is endemic in poor and vulnerable populations for whom access to diagnosis and management may be difficult [8]. When health services are accessed, most children with TB will first present to the same peripheral health facility as other sick children. While health workers in the community and at the primary care level may be equipped to diagnose and manage common childhood illnesses such as acute respiratory infection or diarrhoea, they are less well equipped to detect and diagnose TB in children [9]. Frequently, there is an overlap between the clinical presentation of TB, either as a primary cause or co-morbidity, and the presentation of a number of the more common childhood diseases in these settings $[5,10]$. Smear microscopy for acid-fast bacilli may be available in primary-care settings, but it has limited diagnostic value in young children because of low yield, and chest radiography which is commonly required to support the clinical diagnosis is rarely available in primary-care settings in many TB-endemic areas. In practice, the diagnosis and confirmation of childhood TB will often be limited to secondary or tertiary facilities, either as a primary presentation in the community or by referral from a peripheral health facility, which further increases the challenges of access to care [11-13]. Therefore, at the primary-care level, childhood TB may not be detected early or even at all, resulting in a poor outcome. On the other hand, efforts to increase case detection can readily lead to over-diagnosis, resulting in unnecessary treatment and/or referral with costs to the family and health system.

The WHO guidance on the approach to diagnosing TB in children includes 'bacteriological confirmation whenever possible' [14]. Strengthening the accuracy of diagnosis is important for better patient management and outcome, including of children with drug-resistant $T B$, as well as the quality of the programmatic surveillance data. The WHO classifies TB cases, including child TB cases, for notification and reporting either as 'bacteriologically confirmed' (i.e. a biological specimen is positive by smear microscopy, culture or WHO-approved rapid diagnostics such as Xpert MTB/RIF) or as 'clinically diagnosed' (i.e. not bacteriologically confirmed), which includes cases diagnosed and treated for TB on the basis of radiological abnormalities [14].

Over the last decade, evidence has emerged of the potential feasibility and diagnostic yield of a range of sampling methods as well as tools for bacteriological confirmation [15-19]. This evidence is from a number of TB-endemic countries but is largely from studies of children presenting to tertiary facilities who may be at a more advanced stage of the disease and with a potentially greater diagnostic yield [20]. These research settings also have established laboratory and radiological facilities and staff with expertise and training in diagnosing childhood TB above and beyond that 
which is usually available at the primary-care level in most TB-endemic settings. These diagnostics also require evaluation at the primary-care level where most children with TB will present initially and where the challenges for detection, diagnosis and management may be greatest.

In this issue of the journal, findings are presented from the evaluation of methods for bacteriological confirmation in 119 children aged 2 months to 10 years presenting with TB-related symptoms and signs to a primary-care facility in South Africa [21]. The majority (58\%) of children had a known, current TB case in their household. In addition to clinical evaluation, tuberculin skin test and chest radiograph, samples were collected for bacteriological confirmation of TB including smear microscopy, mycobacterial culture and Xpert MTB/RIF (Xpert) assay. The collection of samples was feasible, and laboratory investigation was completed for a majority of the 469 samples. Successfully tested samples included a single stool sample (for Xpert) from 114 (or 96\%) study participants, a single sputum sample from 14 participants, and, from the 105 who were unable to provide sputum, an induced sputum sample $(n=103)$, at least one nasopharyngeal aspirate $(n=101)$, two nasopharyngeal aspirates $(n=83)$ and gastric aspirate $(n=57)$. Twenty-one (18\%) participants were HIV-infected, 13 of whom provided urine for lipo-arabinomannan assay. Despite the range of samples tested, the diagnostic yield for bacteriological confirmation was very lowfour cases or $3 \%$ overall, and, of these, two were commenced on treatment for TB, one died before treatment was commenced and one was lost-to-follow-up before treatment could be commenced. Treatment for TB was commenced in an additional 46 participants with clinically diagnosed TB and a further 32 were commenced on isoniazid preventive therapy.

Study limitations are acknowledged and, although well recognised for all diagnostic studies in childhood $\mathrm{TB}$, research aiming to improve certainty of diagnosis (or exclusion) of TB in children is central to closing the wide policy-practice gap to improve detection and outcome as well as assessment of a child contact's eligibility for preventive therapy $[7,14]$. A further important consideration is the wider representativeness of the study's findings. The clinical and radiological assessment available at this primary-care level in South Africa may be more comparable to that which is available only at the secondary or tertiary level of care in other TB-endemic settings such as Malawi or Papua New Guinea [13,22]. Furthermore, samples were transported for laboratory testing to a national TB reference laboratory situated less than an hour's drive from the study site, and so there was ready availability of a good laboratory service which would not be accessible in most settings. These are not criticisms of the study but rather a relevant perspective. A low diagnostic yield is reported despite the availability of resources that might be considered optimal and which certainly would not be unavailable in many primary-care settings.

The low yield of bacteriological confirmation reported [21] is not unexpected even in this population with a relatively high pre-test probability of $T B$-a highly TB-endemic setting with a recent TB contact in the majority. Children often have paucibacillary disease, and those presenting as outpatients to a primary-care facility are likely to have less advanced disease with lower potential yield $[20,22]$ than those admitted to a higher level facility. Even in a research setting in a tertiary facility, TB is many children is not bacteriologically confirmed [15-19]. The use of Xpert assay is feasible to confirm diagnosis at the primary-care level; it is more sensitive than smear microscopy and can provide rapid detection of rifampicin resistance. However, Xpert does not detect Mycobacterium tuberculosis in most children with TB. A meta-analysis reported pooled sensitivities for Xpert for sputum and gastric aspirate as $62 \%$ and $66 \%$, respectively, compared with culture, but, given that children with TB are often culture-negative, the sensitivity of Xpert for all TB in children is low [22]. In a recent prospective evaluation at Papua New Guinea's largest paediatric care facility, Xpert assay of sputum or gastric aspirates was positive in only $26(28 \%)$ of 93 inpatient children with presumptive TB [23]. Xpert can not rule out active TB, and this is also important in the evaluation of a child's eligibility for preventive therapy. Therefore, health workers at all levels will continue to rely heavily on clinical evaluation for the detection and management of TB in children, including diagnosis, treatment and prevention.

The decentralisation of child TB services was recently piloted and evaluated in two districts in Uganda, one rural and one urban [11]. Health service strengthening included engagement, training and ongoing mentorship of community and primary-care health workers. The training improved knowledge of diagnosis, including of sputum collection techniques, and the project also provided support to improve clinic and laboratory functionality (e.g. repair of non-functioning microscopes) [11]. Job aids and training tools developed for health workers at the primary or secondary level of care were also employed [24,25]. Evaluation included preand post-implementation comparison of TB programmatic data from within the districts, as well as comparison of child TB notifications in two similar districts where there was no intervention. The main findings were a large increase in child TB notification, including TB in young children, an increase in bacteriologically confirmed TB in children although the majority were diagnosed clinically, an increase in adult TB notification, an improved outcome in children treated for TB and the successful introduction of community-based child contact screening and management with a high uptake and completion of preventive therapy [11]. Efforts are 
ongoing to expand and further evaluate this model in other districts of Uganda as well as in other countries in the region. Furthermore, given the funding challenges of setting priorities in TB control programmes, the evaluation of cost-benefit is important. Nonetheless, it demonstrated that decentralisation of health system capacity is feasible with potential benefits for TB control which reach beyond a focus on children.

Finally, the ability of the health worker at primarycare level to provide optimal care for children presumed to have TB should not be limited to diagnosis. While dependent on many health-care worker and health system-related factors which will vary between local and national settings in TB-endemic countries, important considerations at primary-care level include: (i) the clinical competence and confidence of the health worker to identify the sick child with TB as the primary cause of illness or as a co-morbidity; (ii) the availability of tools to diagnose TB, HIV and malnutrition; (iii) the availability of treatment for TB (including child-friendly treatment formulations), HIV and malnutrition, and preventive therapy for eligible TB contacts; (iv) support for community health workers in the screening and management of TB contacts of all ages, through strengthening TB case detection and provision of preventive therapy for eligible contacts without active TB; and (v) the identification of those who require immediate or non-urgent referral for further diagnosis and/or care. Indications for referral might include: (a) patient requires inpatient facility care on the basis of disease severity such as severe respiratory distress, malnutrition or suspected meningitis; (b) diagnostic work-up requires sampling that cannot be performed at the primary-care level owing to a lack of equipment, lack of training or lack of ability to transfer samples to the laboratory; (c) diagnostic work-up requires other investigations unavailable at the primary-care level, for example, chest radiograph or Xpert assay; (d) drugs unavailable at the primary-care level to treat TB (or co-morbidities such as HIV); and/or (e) presumptive drug-resistant TB for diagnostic work-up and management.

In summary, there is a need to strengthen detection, diagnosis and management of TB in children at the primary-care level to which many children with TB will initially present and where the challenges may be greater than at more central levels of care. The limitations of bacteriological tests highlight the need for training and support in clinical diagnosis and management. It is critical to change the commonly held misconception and dogma that the diagnosis of TB in children is 'always difficult'. A clinical diagnosis can often be made with reasonable accuracy even in a resource-limited context. Developing and strengthening the decentralisation of child TB services will provide important opportunities to potentially improve case detection and treatment outcomes as well as to implement a more family-integrated, community-based model for treatment support and prevention.

\section{ORCID}

S. M. Graham (D) http://orcid.org/0000-0003-3525-2294

\section{References}

[1] World Health Organization. Global tuberculosis report 2018. Geneva: WHO; 2018 [access Feb 1, 2019]. Available from: www.who.int/tb/publications/global_ report/en/

[2] Dodd PJ, Yuen CM, Sismanidis C, et al. The global burden of tuberculosis mortality in children: a mathematical modelling study. Lancet Glob Health. 2017;5:e898-e906.

[3] Marais BJ, Gie RP, Schaaf HS, et al. The natural history of childhood intra-thoracic tuberculosis: a critical review of literature from the pre-chemotherapy era. Int J Tuberc Lung Dis. 2004;8:392-402.

[4] Jenkins HE, Yuen CM, Rodriguez CA, et al. Mortality in children diagnosed with tuberculosis: a systematic review and meta-analysis. Lancet Infect Dis. 2017;17:285-295.

[5] Graham SM, Sismanidis C, Menzies HJ, et al. Importance of tuberculosis control to address child survival. Lancet. 2014;383:1605-1607.

[6] Chiang SS, Khan FA, Milstein MB, et al. Treatment outcomes of childhood tuberculous meningitis: a systematic review and meta-analysis. Lancet Infect Dis. 2014;14:947-957.

[7] World Health Organization/Unicef/Stop TB Partnership/The Union//TAG/KNCV/The Global Fund/ EGPAF/USAID/Unitaid. Roadmap towards ending TB in children and adolescents. Geneva: WHO; 2018 [access Feb 1, 2019]. Available from: www.who.int/ tb/publications/2018/tb-childhoodroadmap/en/

[8] MacPherson P, Khundi M, Nliwasa $M$, et al. Disparities in access to diagnosis and care in Blantyre, Malawi, identified through enhanced tuberculosis surveillance and spatial analysis. BMC Med. 2019;17:21.

[9] Kizito S, Katamba A, Marquez C, et al. Quality of care in childhood tuberculosis diagnosis at primary care clinics in Kampala, Uganda. Int J Tuberc Lung Dis. 2018;22:1196-1202.

[10] Oliwa JN, Karumbi JM, Marais BJ, et al. Tuberculosis as a cause or comorbidity of childhood pneumonia in tuberculosis endemic settings: a systematic review. Lancet Respir Med. 2015;3:235-243.

[11] Zawedde-Muyanja S, Nakanwagi A, Dongo JP, et al. Decentralisation of child tuberculosis services increases case finding and uptake of preventive therapy in Uganda. Int J Tuberc Lung Dis. 2018;11:1314-1321.

[12] Lestari T, Probandari A, Hurtig AK, et al. High caseload of childhood tuberculosis in hospitals on Java Island, Indonesia: a cross sectional study. BMC Public Health. 2011;11:784.

[13] Harries AD, Hargreaves NJ, Graham SM, et al. Childhood tuberculosis in Malawi: nationwide case-finding and treatment outcomes. Int $J$ Tuberc Lung Dis. 2002;6:424-431.

[14] World Health Organization. Guidance for national tuberculosis programmes on the management of 
tuberculosis in children, 2nd edn. Geneva: WHO; 2014 [access Feb 8, 2019]. Available from: www.who.int/tb/ publications/childtb_guidelines/en/

[15] Zar HJ, Hanslo D, Apolles P, et al. Induced sputum versus gastric lavage for microbiological confirmation of pulmonary tuberculosis in infants and young children: a prospective study. Lancet. 2005;365:130-134.

[16] Zar HJ, Workman L, Isaacs W, et al. Rapid molecular diagnosis of pulmonary tuberculosis in children using nasopharyngeal specimens. Clin Infect Dis. 2012;55:1088-1095.

[17] Bates M, O'Grady J, Maeurer M, et al. Assessment of the Xpert MTB/RIF assay for diagnosis of tuberculosis with gastric lavage aspirates in children in sub-Saharan Africa: a prospective descriptive study. Lancet Infect Dis. 2013;13:36-42.

[18] Marcy O, Ung V, Goyet S, et al. Performance of Xpert MTB/RIF and alternative specimen collection methods for the diagnosis of tuberculosis in HIV-infected children. Clin Infect Dis. 2016;62:1161-1168.

[19] Walters E, van der Zalm MM, Palmer $M$, et al. Xpert MTB/RIF on stool is useful for the rapid diagnosis of tuberculosis in young children with severe pulmonary disease. Pediatr Infect Dis J. 2017;36:837-843.
[20] Marais BJ, Hesseling AC, Gie RP, et al. The bacteriologic yield in children with intrathoracic tuberculosis. Clin Infect Dis. 2006;42:e69-e71.

[21] Hanrahan CF, Dansey H, Mutunga L, et al. Diagnostic strategies for childhood tuberculosis in the context of primary care in a high-burden setting: the value of alternative sampling methods. Paediatr Int Child Health. 2019;39.

[22] Detjen AK, Di Nardo AR, Leyden J, et al. Xpert MTB/RIF assay for the diagnosis of pulmonary tuberculosis in children: a systematic review and meta-analysis. Lancet Respir Med. 2015;3:451-461.

[23] Kasa Tom S, Welch H, Kilalang C, et al. Evaluation of Xpert MTB/RIF assay in children with presumed pulmonary tuberculosis in Papua New Guinea. Paediatr Int Child Health. 2018;38:97-105.

[24] International Union Against Tuberculosis and Lung Disease. The Union's desk guide for the diagnosis and management of TB in children. Paris: The Union; 2016 [access Feb 8, 2019]. Available from: www.theu nion.org/what-we-do/publications/technical/deskguide-for-diagnosis-and-management-of-tb-inchildren3

[25] Childhood TB for healthcare workers: an online course. Paris: The Union; 2014 [access Feb 8, 2019]. Available from: https://childhoodtb.theunion.org/courses/CTB1/ en/intro 